\title{
Macroalgas submareales de la bahía de Todos Santos, Baja California, México
}

\section{Submareal macroalgae of the Todos Santos Bay, Baja California, Mexico}

\author{
Raúl Aguilar-Rosas ${ }^{1 *}$, Luis E. Aguilar-Rosas ${ }^{2}$, Guillermo E. Ávila Serrano ${ }^{1}$, Óscar González Yajimovich ${ }^{1}$ y \\ Francisco Becerril Bobadilla ${ }^{1}$
}

${ }^{\prime}$ Facultad de Ciencias Marinas, Universidad Autónoma de Baja California. Km. 107 carretera Tijuana-Ensenada, Ensenada 22830 Baja California, México.

${ }^{2}$ Instituto de Investigaciones Oceanológicas, Universidad Autónoma de Baja California. Km. 107 carretera Tijuana-Ensenada, Ensenada 22830 Baja California, México.

*Correspondencia: aguilarl@uabc.edu.mx

\begin{abstract}
Resumen. Con el fin de identificar y caracterizar la composición de especies de la zona submareal de la bahía de Todos Santos, Baja California, México; se realizaron muestreos de macroalgas marinas bentónicas en 7 sitios durante 19952000. Como resultado de 25 buceos Scuba entre 3 y 33 m de profundidad se encontraron 150 especies de macroalgas para el área de estudio; 10 son Chlorophyta, 26 Phaeophyta y 114 Rhodophyta. Del total, 47 son registros nuevos para el área de estudio y 2 de éstos, Faucheocolax attenuata Setchell y Minium parvum R.L. Moe, son nuevos para la flora marina del Pacífico de México. Se incluye una revisión de las investigaciones en las que se han considerado las macroalgas presentes en la bahía de Todos Santos y una discusión sobre la composición de las especies encontradas, su distribución vertical y reproducción, así como sobre las especies epífitas y parásitas del área.
\end{abstract}

Palabras clave: Chlorophyta, Phaeophyta, Rhodophyta, algas epífitas, registros nuevos, reproducción.

\begin{abstract}
In order to identify and characterize the species composition of the subtidal zone of the Todos Santos Bay, Baja California, Mexico, benthic marine macroalgae were sampled at 7 sites from 1995 to 2000. As a result of 25 scuba at depths between 3 and $33 \mathrm{~m}$ we found a total of 150 species of macroalgae for the study area, of which 10 are Chlorophyta, 26 Phaeophyta and 114 Rhodophyta; 47 species represent new records for the study area, of which Faucheocolax attenuata Setchell and R.L Moe Minium parvum are new to the marine flora of Pacific Mexico. We include a research that consider the macroalgae in the Todos Santos Bay and a discussion on the composition of species found, its vertical distribution and reproduction, particularly the species epiphytes and parasites present in the study area.
\end{abstract}

Key words: Chlorophyta, Phaeophyta, Rhodophyta, epiphytic algae, new records, reproduction.

\section{Introducción}

La costa occidental de Baja California se considera una de las regiones con alta diversidad de especies de macroalgas en el Pacífico de México (Pedroche et al., 1993). Sin embargo, la mayoría de las especies conocidas se ha recolectado en la zona intermareal, y la flora de la zona submareal es poco conocida, debido a los escasos estudios realizados a profundidad (Dawson,1944; Dawson et al., 1960; Guzmán del Proó et al., 1972; Devinny, 1978; Aguilar-Rosas et al.,1990).

Al parecer, los trabajos de Dawson (1944) y Dawson et al. (1960) son los primeros que dan a conocer la

Recibido: 18 mayo 2009; aceptado: 06 noviembre 2010 diversidad de especies en la zona submareal de la costa occidental de Baja California, al describir nuevas especies y ampliar los límites de distribución geográfica en el Pacífico de Norteamérica, y Guzmán del Proó et al. (1972), al determinar la flora macroscópica asociada a los bancos de abulón (Haliotis sp.) en la costa occidental de Baja California, registraron 52 especies en la zona submareal de 19 localidades.

En particular las macroalgas presentes en la bahía de Todos Santos en Baja California han recibido poca atención; los trabajos de Aguilar-Rosas (1981, 1982) y Aguilar-Rosas y Bertsch (1983) indican que la composición de especies de la zona intermareal está integrada por 16 algas verdes (Chlorophyta), 24 pardas (Phaeophyta) y 75 rojas (Rhodophyta). Por otra parte, los escasos registros de especies de macroalgas de la zona submareal provienen de Devinny (1978) quien recolectó 23 especies entre 3 y 
$8 \mathrm{~m}$ de profundidad en Cabo Punta Banda y de AguilarRosas et al. (1990), al registrar un total de 116 especies para 5 sitios de las islas de Todos Santos, 90 de las cuales se encontraron presentes en zona submareal hasta los $24 \mathrm{~m}$ de profundidad.

El objetivo de este trabajo es analizar y dar a conocer la composición de especies de macroalgas de la zona submareal de la bahía de Todos Santos y áreas adyacentes, con muestreos realizados entre 3 y $33 \mathrm{~m}$ de profundidad, proporcionando datos nomenclaturales de las especies, epifitismo, parasitismo y reproducción; asimismo informar sobre la presencia de nuevos registros para el área de estudio y el Pacífico de México.

La bahía de Todos Santos se encuentra localizada en la costa noroccidental de la península de Baja California, entre los $31^{\circ} 41^{\prime}$ y $31^{\circ} 56^{\prime} \mathrm{N}$ y $116^{\circ} 34^{\prime}$ y $116^{\circ} 51^{\prime} \mathrm{O}$ (Fig. 1). Los límites naturales, punta San Miguel al norte, punta Banda al sur y las islas de Todos Santos en la porción central, definen 2 entradas y salidas a la bahía y permiten una circulación continua de agua oceánica (Meillon-Menchaca y Álvarez-Borrego, 1997). Tiene una superficie de 167.6 $\mathrm{km}^{2}$ y una línea de costa compuesta de una ribera rocosa que comprende las puntas San Miguel, El Sauzal, Morro y Ensenada, con playas de bolsillo entre ellas (MeillonMenchaca y Álvarez-Borrego, 1997). Aproximadamente el $90 \%$ de su área se encuentra de 10-50 m de profundidad, el resto forma parte de un angosto canal submarino,

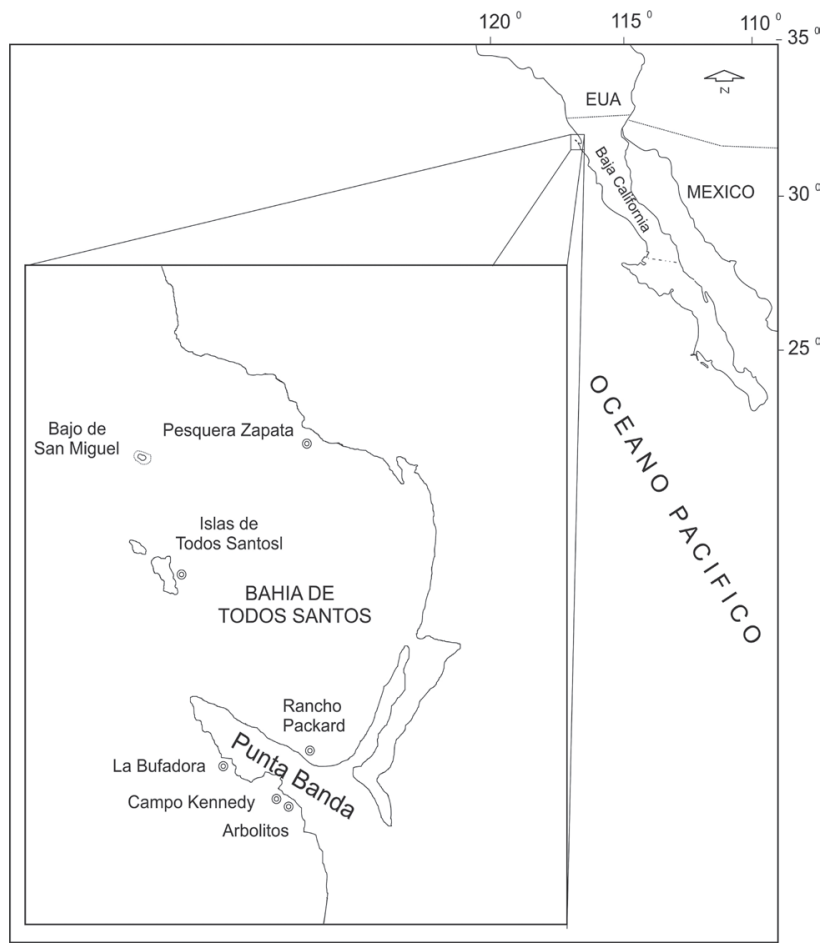

Figura 1. Localización del área de estudio y sitios de muestreo. situado entre punta Banda y las islas de Todos Santos, que alcanzan profundidades de 400 m (Álvarez-Sánchez et al., 1988).

\section{Materiales y métodos}

En el área de estudio se seleccionaron los 7 sitios de muestreo cuyas características se anotan a continuación:

Pesquera Zapata ( $31^{\circ} 51^{\prime} \mathrm{N}$ y $\left.116^{\circ} 41^{\prime} 15^{\prime \prime} \mathrm{O}\right)$. Playa de bolsillo con cantiles de aproximadamente $6 \mathrm{~m}$ de altura; está limitada por salientes rocosas tipo ígneo. La playa es de cantos rodados y en verano predominan arenas, la zona submareal es de pendiente suave con afloramientos rocosos y planicies extensas de arena.

Bajo de San Miguel ( $31^{\circ} 52^{\prime} 30^{\prime \prime} \mathrm{N}$ y $116^{\circ} 45^{\prime} \mathrm{O}$ ). Sitio ubicado un poco al norte de la parte media de la bahía de Todos Santos, entre punta San Miguel y la parte norte de las islas de Todos Santos. Es un montículo rocoso que los pescadores locales nombran "piedra ahogada"; se inicia aproximadamente a los 10 metros y llega a los 32 de profundidad.

Islas de Todos Santos ( $31^{\circ} 48^{\prime} \mathrm{N}$ y $\left.116^{\circ} 48^{\prime} \mathrm{O}\right)$. Estas islas están conformadas por una costa compuesta de cantiles rocosos y playas de cantos rodados donde es accesible el desembarco, excepto en unas pequeñas playas arenosas. La costa este de las islas de Todos Santos está sujeta a un oleaje alto e intenso, mientras que la parte oeste está totalmente protegida. La topografía de la zona submareal es irregular, presenta salientes rocosas entre planicies arenosas que van hasta los $28 \mathrm{~m}$ de profundidad.

Rancho Packard $\left(31^{\circ} 44^{\prime} \mathrm{N}\right.$ y $\left.116^{\circ} 42^{\prime} \mathrm{O}\right)$. Este sitio se caracteriza por presentar cantiles de hasta $20 \mathrm{~m}$ y una playa formada por cantos rodados en la parte superior; disminuye gradualmente su tamaño hacia la parte inferior con salientes de roca ígnea que se extienden hacia la zona submareal, donde sobresalen afloramientos rocosos entre planicies de arena.

La Bufadora ( $31^{\circ} 43^{\prime} 30^{\prime \prime} \mathrm{N}$ y $\left.116^{\circ} 43^{\prime} 15 \mathrm{O}\right)$. Consiste en una costa rocosa limitada hacia el continente por cantiles de hasta $10 \mathrm{~m}$ de alto; presenta una pequeña playa de bolsillo llamada Papalote. Es un área totalmente expuesta al oleaje con una pendiente abrupta y topografía irregular, donde sobresalen grandes montículos rocosos que incluso forman arcos y cuevas bajo la superficie del mar. Además, en el fondo rocoso entre 26 y $30 \mathrm{~m}$ de profundidad destaca una extensa grieta que es un centro hidrotermal producto de la tectónica local propia de la región.

Arbolitos ( $31^{\circ} 42^{\prime} 15^{\prime \prime} \mathrm{N}$ y $116^{\circ} 41^{\prime} 30^{\prime \prime} \mathrm{O}$ ). Este sitio se caracteriza por tener una línea de costa formada de cantiles con altura de hasta $7 \mathrm{~m}$. Su playa está conformada con 
cantos rodados de medianos a grandes, en la parte superior es poco extensa y su tamaño disminuye hacia la base. La limitan salientes rocosas al sur y norte. En la parte media sobresale un gran montículo rocoso que a partir de los 10 $\mathrm{m}$ de profundidad tiene una marcada pendiente. Esta playa está expuesta al fuerte oleaje durante todo el año, ya que su ubicación permite que incida de manera directa el oleaje dominante del noroeste.

Campo Kennedy (. $31^{\circ} 42^{\prime} 30^{\prime \prime} \mathrm{N}$ y $\left.116^{\circ} 41^{\prime} 30^{\prime \prime} \mathrm{O}\right)$. ). Esta línea de costa está conformada por cantiles con una altura promedio de $5 \mathrm{~m}$; la playa es de cantos rodados, y hacia el mar sobresalen afloramientos de rocas entre planicies de arena. Está protegida del oleaje por una saliente rocosa en la parte norte que se extiende al sureste; hacia el sur hay un islote o montículo rocoso con una base ancha desde los $15 \mathrm{~m}$ de profundidad que se angosta en la parte alta ubicada sobre el nivel medio del mar. Es por ello que la playa se utiliza como atracadero de las lanchas de los pescadores.

Durante el periodo comprendido entre agosto de 1995 a julio de 2000, se efectuaron 25 muestreos en los 7 sitios localizados en la bahía de Todos Santos y áreas adyacentes (Cuadro 1). Los ejemplares se recolectaron en la zona submareal entre 3 y $33 \mathrm{~m}$ de profundidad por medio de buceo autónomo Scuba. El material ficológico se colocó en bolsas de polietileno, se etiquetó y se fijó en una solución de formaldehído al $4 \%$ con agua de mar, siendo posteriormente analizado en el laboratorio. Para la descalcificación de algas calcáreas se usó ácido clorhídrico (HCL 1:2). Se realizaron cortes histológicos de las especies y se tiñeron con verde luz y azul de anilina. Con estos cortes se elaboraron preparaciones semipermanentes y se observaron tejidos y estructuras de reproducción útiles para la determinación del material. Las fotografías se tomaron con una

Cuadro 1. Localización de los sitios, con profundidad y fechas de recolecta, en la bahía de Todos Santos y áreas adyacentes, en Baja California, México

\begin{tabular}{|c|c|c|c|}
\hline Sitio & Latitud/longitud & Profundidad & Fecha de recolecta \\
\hline 1. Pesquera Zapata & $\begin{array}{c}31^{\circ} 53^{\prime} 58^{\prime \prime} \mathrm{N} \\
116^{\circ} 43^{\prime} 14^{\prime \prime} \mathrm{O}\end{array}$ & $5 \mathrm{~m}$ & 19 septiembre 1995 \\
\hline 2. Bajo de San Miguel & $\begin{array}{c}31^{\circ} 51^{\prime} 18^{\prime \prime} \mathrm{N} \\
116^{\circ} 48^{\prime} 13^{\prime \prime} \mathrm{O}\end{array}$ & $17 \mathrm{~m}$ & 13 octubre 1995 \\
\hline 3. Islas de Todos Santos & $\begin{array}{c}31^{\circ} 47^{\prime} 30^{\prime \prime} \mathrm{N} \\
116^{\circ} 47^{\prime} 25^{\prime \prime} \mathrm{O}\end{array}$ & $5-27 \mathrm{~m}$ & $\begin{array}{l}25 \text { septiembre } 1995 \\
13 \text { enero } 1996 \\
2 \text { noviembre } 1996 \\
11 \text { mayo } 1997 \\
28 \text { agosto } 1999 \\
11 \text { septiembre } 1999\end{array}$ \\
\hline 4. Rancho Packard & $\begin{array}{l}31^{\circ} 51^{\prime} 36^{\prime \prime} \mathrm{N} \\
116^{\circ} 41^{\prime} 55^{\prime \prime} \mathrm{O}\end{array}$ & $3-5 \mathrm{~m}$ & $\begin{array}{l}18 \text { agosto } 1995 \\
10 \text { octubre } 1996 \\
19 \text { septiembre } 1998\end{array}$ \\
\hline 5. La Bufadora & $\begin{array}{c}31^{\circ} 43^{\prime} 28^{\prime \prime} \mathrm{N} \\
116^{\circ} 433^{\prime} 13^{\prime \prime} \mathrm{O}\end{array}$ & $9-33 \mathrm{~m}$ & $\begin{array}{l}10 \text { agosto } 1995 \\
3 \text { noviembre } 1995 \\
15 \text { septiembre } 1996 \\
7 \text { noviembre } 1996 \\
17 \text { mayo } 1997 \\
28 \text { noviembre } 1999\end{array}$ \\
\hline 6. Campo Kennedy & $\begin{array}{c}31^{\circ} 42^{\prime} 18^{\prime \prime} \mathrm{N} \\
116^{\circ} 40^{\prime} 54^{\prime \prime} \mathrm{O}\end{array}$ & $3-24 \mathrm{~m}$ & $\begin{array}{l}10 \text { agosto } 1995 \\
10 \text { noviembre } 1996 \\
29 \text { noviembre } 1997 \\
31 \text { julio } 1999 \\
26 \text { julio } 2000\end{array}$ \\
\hline 7. Campo Arbolitos & $\begin{array}{l}31^{\circ} 41^{\prime} 44^{\prime \prime} \mathrm{N} \\
116^{\circ} 40^{\prime} 39^{\prime \prime} \mathrm{O}\end{array}$ & $12-31 \mathrm{~m}$ & $\begin{array}{l}19 \text { noviembre } 1995 \\
3 \text { agosto } 1996 \\
16 \text { septiembre } 1996\end{array}$ \\
\hline
\end{tabular}


cámara digital (Sony DSC-S85, Tokyo, Japan) conectada a un microscopio Zeiss (Axioscop 40, Goettingen, Germany). Las figuras fueron editadas usando el programa Adobe ${ }^{\circledR}$ Photoshop ${ }^{\circledR} 7.0$ (Adobe Systems Inc., San Jose, USA).

Para determinar el material ficológico se utilizaron los trabajos de Dawson (1953, 1954, 1960, 1961, 1962, 1963a, 1963b); Abbott y Hollenberg (1976); Gabrielson et al. (1989). La nomenclatura se actualizó de acuerdo con Silva et al. (1996); Stewart (1991), Scagel et al. (1989), Miller (2009), Pedroche et al. (2005), Pedroche et al. (2008). La lista de especies se elaboró, siguiendo el arreglo sistemático y la clasificación establecidos por Scagel et al. (1989), Silva et al. (1996), Pedroche et al. (2005), Pedroche et al. (2008). El material fue procesado y depositado en el herbario CMMEX de la Facultad de Ciencias Marinas, de la Universidad Autónoma de Baja California (Holmgren et al.,1990).

\section{Resultados}

Composición florística y nuevos registros. Para la zona submareal de la bahía de Todos Santos (3-33 m) se determinaron 150 especies de algas marinas bentónicas, epífitas o parásitas; de las cuales 114 (76 \%) corresponden a Rhodophyta, $26(17 \%)$ a Phaeophyta y $10(7 \%)$ a Chlorophyta (Apéndice).

La división Rhodophyta dominó en términos de diversidad, seguida en orden de importancia por Phaeophyta y Chlorophyta. La familia de la división Rhodophyta mejor representada en especies fue Corallinaceae con 22 taxa, de las cuales sobresalen 3 de Corallina y 3 de Lithophyllum. Sigue en importancia Ceramiaceae con 20 especies, donde destacan Antithamnion y Ceramium con 4 especies cada uno; mientras que para Rhodomelaceae con 15 taxa, se ubican Laurencia con 3 especies, Polysiphonia y Pterosiphonia con 2 especies cada uno.

Con respecto a las Phaeophyta, las familias mejor representadas fueron Scytosiphonaceae y Dictyotaceae con 5 especies cada una, de las cuales destacan 3 del género Colpomenia y 2 de Dictyota. Finalmente, en la División Chlorophyta sobresalen las familias Codiaceae con 4 especies de Codium y Ulvaceae con 3 de Ulva.

En este estudio se identificaron 150 especies, de las cuales 47 (31\%) son nuevos registros para la bahía de Todos Santos y de éstas,. Faucheocolax attenuata Setchell (Fig. 2) y Minium parvum R. L Moe (Fig. 3) se registran por primera vez para la costa del Pacífico de México (Apéndice). Cabe señalar que del total, 65 son epífitas y 2 parásitas, lo que corresponde al $44.6 \%$ de especies (Apéndice), 53

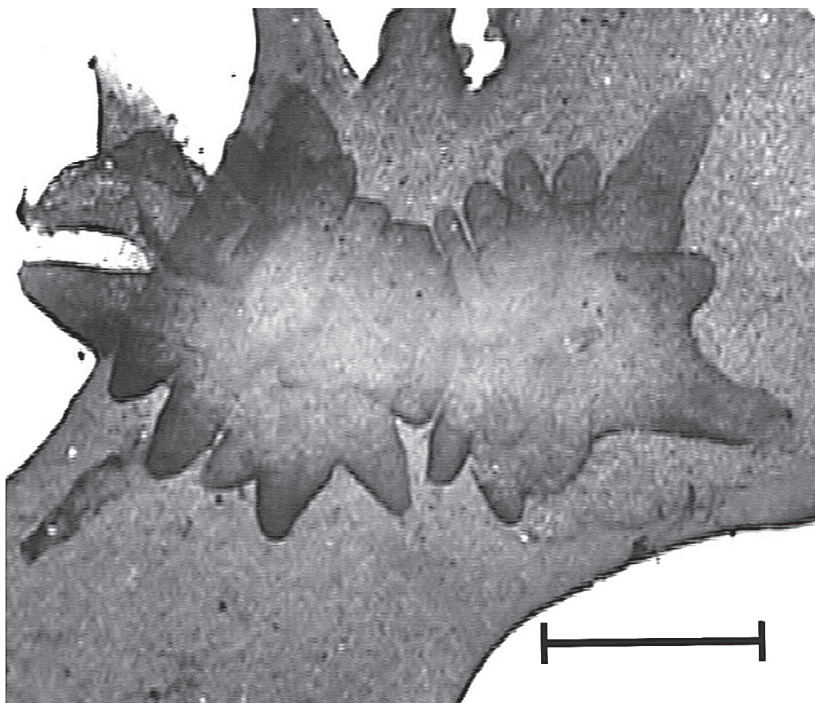

Figura 2. Hábito del talo Faucheocolax attenuata Setchell (escala: $1 \mathrm{~mm}$ ).
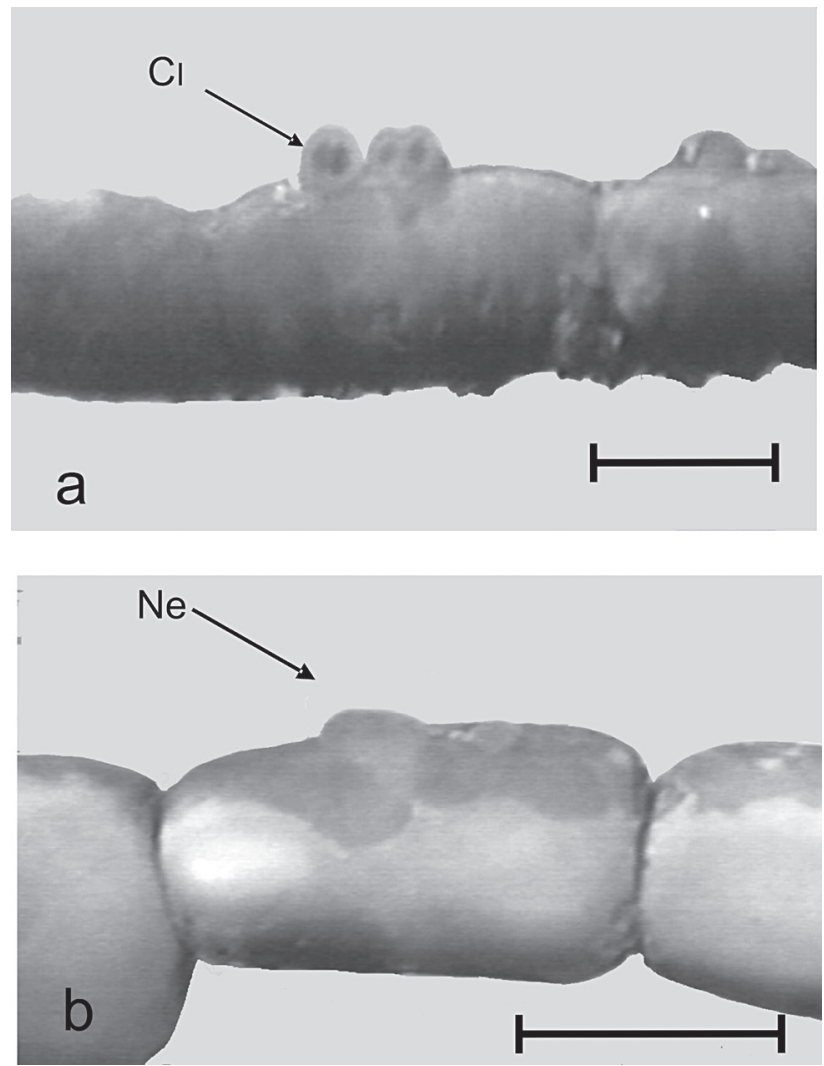

Figura 3. Minium parvum Moe. a) hábito del talo cistocárpico $($ escala $=2 \mathrm{~mm})$; b) hábito del talo esporangial (escala $=3 \mathrm{~mm}$ ).

$\mathrm{Ci}=$ Cistocarpo. $\mathrm{Ne}=$ nematecio. 
corresponden al grupo Rhodophyta, 7 a Phaeophyta y 7 a Chlorophyta. Se observó un incremento en el número de taxa con esta forma de vida en las especies de las familias Ceramiaceae y Rhodomelacea (Rhodophyta).

La mayoría de las epífitas que fueron encontradas crecen sobre una gran variedad de algas, sin preferencia alguna; destacan Acrosorium uncinatum, Ceramium eatonianum, Colaconema garbaryi, Cryptopleura lobulifera, Herposiphonia verticillata, Heterosiphonia erecta, Microcladia coulteri y Pterosiphonia dendroidea. Por el contrario, algunas de las especies epífitas o parásitas mostraron especificidad por algún hospedero; tal es el caso de Ceramium sinicola sobre Codium, Erythrocystis saccata sobre Laurencia, Streblonema transfixum sobre Desmarestia munda, Jantinella verrucaeformis parásita en Chondria acrorhyzophora, Faucheocolax attenuata parásita en Gloiocladia laciniata y las algas rojas Melobesia mediocris y Smithora naiadum sobre las hojas del pasto marino Phyllospadix torreyii.

Las algas que sobresalen por presentar un número alto de epífitas o que son utilizadas como sustrato son las coralinas articuladas (Bossiella, Calliarthron, Corallina), las especies de los géneros Chondracanthus, Gelidium, Codium, Laurencia y el alga parda introducida Sargassum muticum.

En las especies determinadas predominó el tipo de reproducción asexual con la presencia de talos monosporangiales, bisporangiales y tetrasporangiales, en el grupo de algas rojas; seguidas por plantas cistocárpicas, y con menos frecuencia por las gametangiales masculinas. En algunas se presentaron todas las fases reproductivas con plantas tetraspóricas, cistocárpicas y gametofitas masculinas, en la misma localidad, como fue el caso de Antithamnion kylinii, Dasya sinicola, Ptilothamnionopsis lejolisea, Cryptopleura peltata, Polysiphonia pacifica y Pterosiphonia dendroidea.

La mayoría de las Phaeophyta se observaron en estado vegetativo; sin embargo, en algunas especies se presentaron plantas gametofíticas masculinas con anteridios y femeninas con oogonios, como fue el caso de Dyctiopteris undulata, Dictyota binghamiae, Dictyota coriacea, Cystoseira osmundacea, Halidrys dioica y Sargassum muticum. Menos frecuentes fueron las esporofíticas con esporangios uniloculares y pluriloculares. En cuanto a las Chlorophyta, a excepción de Codium fragile que presentó gametangios, el resto de las especies identificadas se observaron en estado vegetativo.

En el Cuadro 2 se muestra el análisis de la distribución vertical de las especies en relación con un incremento cada $5 \mathrm{~m}$. Para las 3 divisiones de macroalgas analizadas hay un marcado incremento en el número de especies hasta una profundidad de 5-10 $\mathrm{m}$ de profundidad; a partir de este intervalo es notorio observar que el número de especies decrece gradualmente al incrementarse la profundidad entre los 30 y $35 \mathrm{~m}$. En el intervalo de $25-30 \mathrm{~m}$ de profundidad se presentan 28 especies $(6.42 \%)$, de las cuales destaca el grupo de algas rojas integrado por especies de Bossiella, Calliarthron, Lithothamnium y Yamadaia; Chondracanthus corymbiferus, Callophyllis flabellulata, Pikea robusta, Ozophora clevelandii, Fryeella gardneri, Rhodymenia callophyllidoides, Veleroa subulata; y el alga verde Codium hubbsii (véase Apéndice). Sólo el $0.68 \%$ de las especies se encontró creciendo por debajo de los $30 \mathrm{~m}$ de profundidad, representado por las algas rojas Pterothamnion villosum y Heterosiphonia erecta, así como el alga parda Desmarestia tabacoides (Cuadro 2). Del total de las especies identificadas, solamente 42 (28\%) muestran una amplia distribución vertical entre los 3 y $20 \mathrm{~m}$ de profundidad (Apéndice).

\section{Discusión}

El número total de especies que se encontraron en la bahía de Todos Santos (150) supera el hallado en estudios previos realizados en la zona submareal en la costa occidental de Baja California (Dawson, 1944; Dawson et al., 1960; Aguilar-Rosas et al., 1990). En estudios previos realizados en la bahía y áreas adyacentes se informa de la presencia de 138 especies de macroalgas recolectadas entre 3 y 24 m de profundidad (Guzmán del Proó et al., 1972; Aguilar-Rosas et al., 1990; Devinny, 1978).

En el presente estudio se encontraron las 17 especies de macroalgas asociadas a los bancos de abulón (Haliotis spp.) que Guzmán del Proó et al. (1972) registraron para algunas localidades dentro de la bahía de Todos Santos; de la misma manera, 67 de las 70 citadas por Devinny (1978) para cabo Punta Banda y 77 de las 107 registradas para la comunidad de algas submareales en las islas de Todos Santos por Aguilar-Rosas et al. (1990). Posiblemente las especies no localizadas quedaron excluidas por el tipo de muestreo que se utilizó en este trabajo, por su estacionalidad, o porque debido a su pequeño tamaño pasaron desapercibidas.

Cabe mencionar que en el periodo posterior al muestreo del presente estudio se registraron 2 especies de gran interés para la ecología de la zona submareal, por considerarse introducidas e invasoras para la costa de Baja California, México: Undaria pinnatifida Okamura, que comúnmente se encontró creciendo sobre rocas desde la zona intermareal hasta $20 \mathrm{~m}$ de profundidad, en las islas de Todos Santos (Aguilar-Rosas et al., 2005), y Sargassum filicinum, desde la zona intermareal hasta $6 \mathrm{~m}$ de profundi- 
Cuadro 2. Análisis de la distribución vertical de las algas submareales de la bahía de Todos Santos, Baja California, usando un incremento cada 5 metros

\begin{tabular}{|c|c|c|c|c|c|c|c|c|}
\hline \multirow[t]{2}{*}{ Intervalo de profundidad } & \multicolumn{2}{|c|}{ Rhodophyta } & \multicolumn{2}{|c|}{ Phaeophyta } & \multicolumn{2}{|c|}{ Chlorophyta } & \multicolumn{2}{|c|}{ Total } \\
\hline & $s p p$. & $\%$ & spp. & $\%$ & spp. & $\%$ & spp. & $\%$ \\
\hline $1-5 \mathrm{~m}$ & 59 & 17.82 & 16 & 20.25 & 5 & 19.23 & 80 & 18.34 \\
\hline $5-10 \mathrm{~m}$ & 74 & 22.35 & 18 & 22.78 & 7 & 26.92 & 99 & 22.49 \\
\hline $10-15 \mathrm{~m}$ & 62 & 18.73 & 17 & 21.51 & 6 & 23.07 & 85 & 19.49 \\
\hline $15-20 \mathrm{~m}$ & 64 & 19.33 & 15 & 18.98 & 5 & 19.23 & 84 & 18.34 \\
\hline $20-25 \mathrm{~m}$ & 47 & 14.19 & 8 & 10.12 & 2 & 7.69 & 57 & 13.07 \\
\hline $25-30 \mathrm{~m}$ & 23 & 6.94 & 4 & 5.06 & 1 & 3.84 & 28 & 6.42 \\
\hline $30-35 \mathrm{~m}$ & 2 & 0.60 & 1 & 1.26 & 0 & 0.00 & 3 & 0.68 \\
\hline
\end{tabular}

dad en Rancho Packard (Aguilar-Rosas et al., 2007).

El número total de especies registradas durante el estudio es relativamente alto comparado con inventarios conocidos para el sur de California (Abbott y Hollenberg, 1976; Devinny y Kirkwood, 1974; Breda y Foster, 1985), lo que sugiere que la bahía de Todos Santos posee una riqueza de especies muy alta y que la composición de las mismas hace de este lugar de tan variada y abundante flora marina, típica de las regiones templadas, un sistema ecológico de importancia (Murray et al., 1980).

Las especies registradas en este trabajo se encuentran ampliamente distribuidas en California (Devinny y Kirkwood, 1974; Breda y Foster, 1985); para la zona submareal se han identificado algunas especies indicadoras de aguas cálidas, como Codium cuneatum, Dictyopteris undulata, Hydroclathrus clathratus, Zonaria farlowii, Gelidium nudifons y Lithotrix aspergillum; además, especies propias de aguas frías, como Desmarestia munda, Laminaria setchellii, Grateloupia americana, Nienburgia andersoniana, Opuntiella californica, Callophyllis flabellulata y Chondracanthus corymbiferus (Murray et al., 1980; Engle, 1994), las cuales también forman parte de la flora algal submareal de la bahía de Todos Santos, posiblemente como un reflejo de las características fisicoquímicas que tienen en común y la presencia del fenómeno de surgencias (Dawson, 1951).

Las rodofíceas Faucheocolax attenuata y Minium parvum son registros nuevos para Baja California, en particular F. attenuata, parásita en especies de Gloiocladia, que crece en la zona submareal hasta $10 \mathrm{~m}$ de profundidad; su distribución geográfica conocida es la costa del Pacífico de Norteamérica, desde Columbia Británica hasta Carmel Bay, California, que es la localidad tipo (Scagel et al., 1989; Setchell, 1923, Abbott y Hollenberg, 1976). Los talos tetraspóricos y cistocárpicos de $F$. attenuata que se encontraron en Arbolitos y La Bufadora, Baja California, como parásitos de Gloiocladia laciniata, entre 9 y $12 \mathrm{~m}$ de profundidad, presentan las mismas características morfológicas y reproductivas descritas e ilustradas para ejemplares recolectados en California (Setchell, 1923, Abbott y Hollenberg, 1976). Con base en el material recolectado, se amplía aproximadamente en $600 \mathrm{~km}$ el límite sur de distribución geográfico de F. attenuata en el Pacífico de Norteamérica, desde Carmel Bay, California, hasta Cabo Punta Banda, Baja California.

Minium parvum representa el caso de alga roja costrosa, no calcárea, que crece epífita en especies de algas articuladas coralinas de los géneros Calliarthron, Bossiella, Serraticardia y Corallina; originalmente fue descrita con material recolectado entre 5 y $25 \mathrm{~m}$ de profundidad en Farallon Islands y Carmel Bay en la costa central de California (Moe, 1979). Otras poblaciones conocidas se localizan en isla Vancouver, Columbia Británica (Garbary et al., 1984; Hawkes y Scagel, 1986). La presencia de 
plantas tetraspóricas y cistocárpicas de $M$. parvum en la bahía de Todos Santos, Baja California, muestran que esta especie está perfectamente adaptada a su hábitat entre 5 y $27 \mathrm{~m}$ de profundidad. Posiblemente su carácter epífito, costroso y su pequeño tamaño no permitieron localizarla con anterioridad en el área de estudio, a pesar de que se han citado un gran número de especies de algas calcáreas entre 6 y 9 m de profundidad, como B. orbigniana, Calliarthron cheilosporioides, Corallina officinalis, Jania tenella y Lithothrix aspergillum (Aguilar-Rosas et al., 1990), consideradas hospederos potenciales de M. parvum. Las plantas que se encontraron presentan las mismas características morfológicas y reproductivas descritas e ilustradas para ejemplares recolectados en California y Columbia Británica (Moe, 1979; Hawkes y Scagel, 1986). Con el material recolectado se amplía aproximadamente $600 \mathrm{~km}$ el límite sur de distribución geográfico de M. parvum en el Pacífico de Norteamérica desde Carmel Bay, California hasta Cabo Punta Banda, Baja California.

En el área de estudio las especies de algas se desarrollan de preferencia sobre un sustrato rocoso; sin embargo, se encontraron creciendo epífitas o parásitas cerca del $44.7 \%$ (67 especies). La relación simbiótica más común que se presenta entre las algas marinas es crecer como epífitas o parásitas en otras plantas (algas, pastos marinos y raíces de mangle; Goff y Coleman, 1985). En algunos casos, la presencia de las epífitas en un hospedante puede ser fortuita, esto es, se comportan como especies oportunistas y se sirven de una gran variedad de hospedantes (facultativas); en otros casos, existe un alto grado de especificidad (obligadas), y se presentan in situ sólo en un número limitado de hospedantes, lo que es particularmente evidente en las algas parásitas rojas que frecuentemente están restringidas a una sola especie hospedante (Goff, 1983; Goff y Coleman, 1985). Las algas coralinas articuladas, en combinación con especies de Chondracanthus, Gelidium, Laurencia, Codium y el alga parda Sargassum muticum, desempeñan un papel importante al servir como estructura de soporte o sustrato para un gran número de algas epífitas o parásitas en la zona submareal, siendo la mayoría especies muy pequeñas. Tales resultados también se encontraron en estudios florísticos realizados en las costas de Baja California y California (Murray y Littler, 1989; Murray y Horn, 1989; Aguilar-Rosas et al., 1990; Aguilar-Rosas y Aguilar-Rosas, 1994; Mateo-Cid y Mendoza-González, 1994).

La duración y permanencia de las especies en la zona submareal de la bahía de Todos Santos posiblemente está relacionada con el tipo de reproducción (vegetativa, asexual y sexual) y el ciclo de vida de las especies, ya que en todos los sitios se encontraron talos en reproducción y para algunas especies de algas rojas se observaron las
3 fases reproductivas (tetrasporofitos, carposporofitos y gametofitos masculinos) en el mismo sitio (Apéndice). El número relativo y predominancia de talos en estado reproductivo o vegetativo que se ha señalado, varía de acuerdo con los cambios de salinidad, temperatura, tipo de sustrato, el tipo de ciclo reproductivo y supervivencia de esporas (Schiel y Foster, 1986; Nelson, 1989). La mayoría de las algas pardas y verdes se encontraron en estado vegetativo, posiblemente debido a la estacionalidad de las especies o a que los ejemplares recolectados estaban en estadios juveniles y no tenían desarrolladas sus estructuras reproductivas $\mathrm{y}$, además, a que los talos gametofitos masculinos son poco frecuentes y de vida corta (Santelices, 1977; Littler et al., 1983; Hawkes, 1990).

La totalidad de las especies identificadas en este estudio también se registran para la zona submareal somera (<30 m) de California (Abbott y Hollenberg, 1976), y aproximadamente el $50 \%$ de las especies que forman parte de la comunidad algal en la zona submareal profunda, entre los 30 y $80 \mathrm{~m}$ de profundidad para la parte central de California (Spalding et al., 2003), se encuentran presentes en la bahía de Todos Santos.

En este estudio se determinó que el número de especies decrece al aumentar la profundidad; este mismo patrón fue descrito para la flora submareal de las islas Todos Santos, Baja California (Aguilar-Rosas et al., 1990), la isla Oki, Japon (Kajimura, 1987) y la isla Daedo, Korea (Kim et al., 1996).

Tomando en cuenta la composición de especies que fueron encontradas en la zona submareal, se observa que la estructura de la comunidad está integrada por algas pardas formadoras de extensas praderas o mantos, como es el caso de Egregia menziesii, Eisenia arborea, Laminaria setchellii, Macrocystis pyrifera y Sargassum muticum, sobre todo en los sitios localizados en cabo Punta Banda y las islas de Todos Santos. Además, en el fondo se define una comunidad integrada por algas rojas foliosas, coralinas articuladas y una fina capa de algas filamentosas y costrosas. Esta estructura de la comunidad se ha descrito para la flora submareal de Baja California (Dawson et al., 1960; Aguilar-Rosas et al., 1990) y California (Allen, 1973; Devinny y Kirkwood, 1974; Lewbel et al., 1981; Lissner, 1986; Breda y Foster, 1985; Konar y Foster, 1992; Konar y Roberts, 1996).

En conclusión, el análisis de la flora algal marina de la zona submareal de la bahía de Todos Santos, indica que este cuerpo de agua cuenta con una elevada riqueza de especies con comunidades de macroalgas bien desarrolladas entre 3 y $33 \mathrm{~m}$ de profundidad que crecen sobre fondo rocoso, sobresaliendo un gran número de especies epífitas, así como la presencia de grandes algas pardas típicas de una zona templada. Lo anterior sugiere la necesidad de conti- 
nuar con estudios en otras localidades de Baja California, a fin de ampliar el conocimiento sobre la flora de aguas profundas y poder realizar un análisis biogeográfico de las especies a lo largo de la costa del Pacífico de México.

\section{Agradecimientos}

A la Facultad de Ciencias Marinas e Instituto de Investigaciones Oceanológicas de la Universidad Autónoma de Baja California, por el apoyo económico brindado para la realización del presente trabajo (Programa 243). A Marcela Morales, Germán Pérez Plascencia, Héctor Lizarraga Cubedo y José Pech Pacheco, por su ayuda en la recolecta del material. A Paul C. Silva y Richard L. Moe del Herbario de la Universidad del Sur de California (UC) en Berkeley, por la literatura brindada, la corroboración de algunas especies y permitirnos la consulta del material de herbario.

\section{Literatura citada}

Abbott, I. A. y G. J. Hollenberg. 1976. Marine algae of California. Stanford University Press, California. $827 \mathrm{p}$.

Aguilar Rosas, L. E. 1981. Algas rojas (Rhodophyta) de la bahía Todos Santos, Baja California, Mexico durante el ciclo anual 1978-1979. Ciencias Marinas 7:85-101.

Aguilar Rosas, L. E. 1982. Ocurrencia de algas cafés (Phaeophyta) en la bahía Todos Santos, Baja California. Ciencias Marinas 8:25-34.

Aguilar Rosas, L. E., y H. Bertsch. 1983. Algas verdes (Chlorophyta) de la bahía Todos Santos, Baja California, México. Ciencias Marinas 9:111-123.

Aguilar-Rosas, L. E., R. Aguilar-Rosas, H. Hawai, S. Uwai y E. Valenzuela-Espinoza. 2007. New record of Sargassum flicinum Harvey (Fucales, Phaeophyceae) in the Pacific coast of Mexico. Algae 22:17-21.

Aguilar-Rosas, R., I. Pacheco-Ruiz y L. E. Aguilar-Rosas. 1990. Algas marinas de las islas Todos Santos, Baja California, México. Marine algae from the Todos Santos Islands, Baja California, México. Ciencias Marinas 16:117-129.

Aguilar-Rosas, R. y M. A. Aguilar-Rosas. 1994. Estudio florístico de las algas marinas bentónicas del ejido San José, Baja California, México. Ciencias Marinas 20:511-534.

Aguilar-Rosas, R., L. E. Aguilar-Rosas, G. Ávila-Serrano y R. Marcos-Ramírez. 2004. First record of Undaria pinnatifida (Harvey) Suringar (Laminariales, Phaeophyta) on the Pacific coast of Mexico. Botanica Marina 47:255-258.

Aguilar-Rosas, R., L. E. Aguilar-Rosas y F. Pedroche. 2004. Ulva fascista Delile (Ulvaceae, Chlorophycota): a species newly introduced into Pacific Mexico. Botanica Marina 48:46-51.

Álvarez-Sánchez, L. G., R. Hernández-Walls y R. DurazoArvizu. 1988. Patrones de deriva de trazadores lagrangianos en la bahía de Todos Santos. Ciencias Marinas 14:135-162.

Aleen, A. A. 1973. Ecology of a kelp-bed in Southern California. Botanica Marina 16:83-95.

Breda, V. A. y Foster, M. S., 1985. Composition, abundance, and phenology of foliose red algae associated with two central California kelp forests. Journal of Experimental Marine Biology and Ecology 94:115-130.

Dawson, E. Y. 1944. Some new and unreported sublittoral algae from Cerros I., Mexico. Bulletin Southern California Academy of Science 43:102-112.

Dawson, E. Y. 1951. A further study of upwelling and associated vegetation along Pacific Baja California, Mexico. Journal of Marine Research 10:39-58.

Dawson, E. Y. 1953. Marine red algae of Pacific Mexico. I. Bangiales to Corallinaceae subf. Corallinoideae. Allan Hancock Pacific Expeditions 17:1-239.

Dawson, E. Y. 1954. Marine red algae of Pacific Mexico. II. Cryptonemiales. Allan Hancock Pacific Expeditions 17: 241-397.

Dawson, E. Y. 1960. Marine red algae of Pacific Mexico. III. Cryptonemiales. Corallinaceae subf. Melobesioideae. Pacific Naturalist 2:1-25.

Dawson, E. Y. 1961. Marine red algae of Pacific Mexico. IV. Gigartinales. Pacific Naturalist 2:191-341.

Dawson, E. Y. 1962. Marine red algae of Pacific Mexico. VII. Ceramiales, Ceramiaceae, Delesseriaceae. Allan Hancock Pacific Expeditions 26:1-207.

Dawson, E. Y. 1963a. Marine red algae of Pacific Mexico. Part VI. Rhodymeniales. Nova Hedwigia 5:437-476.

Dawson, E. Y. 1963b. Marine red algae of Pacific Mexico. Part VIII. Ceramiales: Dasyaceae, Rhodomelaceae. Nova Hedwigia 6:401-481.

Dawson, E. Y., Neushul, M. y R. Wildman. 1960. New records of sublittoral marine plants from Pacific Baja California. Pacific Naturalist 1:1-30.

Devinny, J. S. 1978. Ordination of seaweed communities: Environment gradients at Punta Banda, Mexico. Botanica Marina 21:357-363.

Devinny, J. S. y P. D. Kirkwood. 1974. Algae associated with kelp beds of the Monterey Peninsula, California. Botanica Marina 17: 100-106.

Gabrielson, P. W., R. F. Scagel y T. B. Widdowson. 1989. Key to the benthic marine algae and seagrasses of British Columbia, Southeast Alaska, Washington and Oregon. Phycological Contribution No. 4, University of British Columbia, Vancouver. $187 \mathrm{p}$.

Garbary, D. J., G. I. Hansen y R. F. Scagel. 1984. Additions to the algae of Barkley Sound, Vancouver Island, British Columbia. Syesis 17:43-45.

Goff, L. J. y A. W. Coleman. 1985. The role of secundary pit 
connections in red algal parasitism. Journal of Phycology 21:483-508.

Guzmán del Proó, S. A., S. de la Campa de Guzmán y J. Pineda Barrera. 1972. Flora macroscópica asociada a los bancos de abulón (Haliotis sp.) en algunas áreas de la costa occidental de Baja California. Memorias del IV Congreso Nacional de Oceanografía, 17-19 de noviembre, 1969, Instituto de Ciencias del Mar y Limnología, UNAM, México, D.F., p 257-263.

Engle, J. M. 1994. Perspectives on the structure and dynamics of nearshore marine assemblages of the California Channel Islands. In The Fourth California Islands Symposium: Update on the Status of Resources, W.L. Halvorson and G.J. Maender (eds.) Santa Barbara Museum of Natural History, California. p. 13-23.

Hawkes, M. W. 1990. Reproductive strategies. In Biology of the red algae, K. M. Cole and R. G. Sheat (eds.) Cambridge University Press. p. 55-476.

Hawkes, M. W. y R. F. Scagel. 1986. The marine algae of British Columbia and northern Washington: Division Rhodophyta (red algae), class Rhodophyceae, order Rhodymeniales. Canadian Journal of Botany 64:1549-1580.

Holmgren, P. K., N. H. Holmgren y I. C. Barnett. 1990. Index Herbariorum, Pt 1: The Herbaria of the World, 8th ed. [Regnum Vegetabile, vol. 20]. New York Botanical Garden, Bronx, New York, $\mathrm{x}+693 \mathrm{p}$.

Kajimura, M. 1987. Deep-water flora of benthic marine algae in the Oki Islands, Sea of Japan. Botanica Marina, 30:373-385.

Kim, K. Y., S. H. Huh y G. H. Kim. 1996. Diversity and abundance of sublittoral macroalge around Daedo Island, the South coast of Korea. Algae 11:171-177.

Konar, B. y M. S. Foster. 1992. Distribution and recruitment of subtidal geniculate coralline algae. Journal of Phycology 28:273-280.

Konar, B. y C. Roberts. 1996. Large scale landslide effects on two exposed rocky subtidal areas in California. Botanica Marina 39:517-524.

Lewbel, G. S., A. Wolfson, T. Gerrodette, W. H. Lippincott, J. L. Wilson y M. M. Littler. 1981. Shallow-water benthic communities on California's outer continental shelf. Marine Ecology Progress Series 4:159-168.

Lissner, A. L. 1986. Deep-water biological assemblages of a hard-bottom bank-ridge complex of the Southern California Continental borderland. Bulletin Southern California Academy of Science 85:87-101.

Littler, M. M., D. R. Martz y D. S. Littler. 1983. Effects of recurrent sand deposition on rocky intertidal organisms: importance of substrate heterogeneity in a fluctuating environment. Marine Ecology Progress Series 11:129-139.

Mateo-Cid, L. E. y A. C. Mendoza-González. 1994. Estudio florístico de las algas bentónicas de bahía Asunción, Baja California Sur, México. Ciencias Marinas 20: 41-64.

Meillon-Menchaca, O. y J. Álvarez-Borrego. 1997. Análisis del comportamiento espacial del oleaje en la bahía de Todos Santos, Baja California, mediante imágenes de superficies marinas. Ciencias Marinas 23:23-41.

Miller, K. A. 2009. Seaweeds of California. ucjeps.berkeley.edu/ californiaseaweeds.html. UC-JEPS: Updates of California Seaweeds Species List. 39 p.

Moe, R. L. 1979. Minium parvum gen. et sp. nov., a crustose member of the Rhodymeniales (Rhodophyta). Phycologia 18:38-46.

Murray, S. N. y M. H. Horn. 1989. Seasonal dynamics of macrophyte populations from an Eastern North Pacific rocky-intertidal habitat. Botanica Marina 32:41-51.

Murray, S. N. y M. M. Littler. 1989. Seaweeds and seagrasses of Southern California: distributional lists for twenty-one rocky intertidal sites. Bulletin Southern California Academy of Science 88:61-79.

Nelson, W. A. 1989. Phenology of Gracilaria sordida W. Nelson populations. Reproductive status, plant and population size. Botanica Marina 32:41-51.

Okamura, K. 1908. Icones of Japanese Algae, vol. I Tokyo. p. 179-208, pls. XXXVI-XL.

Pedroche, F. F., K. M. Dreckemann, A. G. Sentíes y R. MargainHernández. 1993. Diversidad algal en México. Revista de la Sociedad Mexicana de Historia Natural 44:69-92.

Pedroche, F. F., P. C. Silva, L. E. Aguilar-Rosas, K. M. Dreckmann y R. Aguilar-Rosas. 2005. Catálogo de las algas marinas bentónicas del Pacífico de México. I. Chlorophycota. Universidad Autónoma de Baja California, Ensenada. p. i-viii, 17-146.

Pedroche, F. F., P. C. Silva, L. E. Aguilar-Rosas, K. M. Dreckmann y R. Aguilar-Rosas. 2008. Catálogo de las algas bentónicas del Pacífico de México II. Phaeophycota. Universidad Autónoma Metropolitana, México, D.F./ University of California, Berkeley. p. [i-viii], i-vi, 15-146.

Peters, A. F., M. J. H. van Oppen, C. A Wiencke, W. T Stam y J. L. Olsen. 1997. Phylogeny and historical ecology of the Desmarestiaceae (Phaeophyceae) support a southern hemisphere origin. Journal of Phycology 33:294-309.

Santelices, N. 1977. Ecología de las algas marinas bentónicas. Universidad Católica de Chile, Santiago. 384 p.

Scagel, R. F. 1957. An annotated list of the marine algae of British Columbia and northern Washington. National Museum of Canada, Bulletin 150:1-289.

Scagel, R. F., P. W. Gabrielson, D. G. Garbary, L. Golden, M. W. Hawkes, S. C. Lindstrom, J. C. Oliveira y T. B. Widdowson. 1989. Sinopsis of the benthic marine algae of British Columbia, Southeast Alaska, Washington and Oregon. Phycological Contribution No.3, University of British Columbia, Vancouver. 532 p.

Schiel, D. y M. S. Foster. 1986. The structure of subtidal algal stands in temperate waters. Oceanography and Marine Biology Annual Review 24:481-489.

Setchell, W. A. 1923. Parasitic Florideae, II. University of 
California Publications in Botany 10:393-396.

Silva, P. C., P.W. Basson y R.L. Moe. 1996. Catalogue of benthic marine algae of the Indian Ocean. University of California Publications in Botany 79:1-259 p.

Spalding, H., M. S. Foster y J. N. Heine. 2003. Composition, distribution, and abundance of deep-water $(>30 \mathrm{~m})$ macroalgae in central California. Journal of Phycology $39: 273-284$
Stewart, J. G. 1991. Marine Algae and seagrasses of San Diego County. California Sea Grant College, La Jolla, California. $197 \mathrm{p}$.

Yoshida, T. 1998. Marine algae of Japan. Uchida Rokakuho Publishing. p. $25+1222$. Tokyo.

Wynne, M. J. 1985. Nomenclatural assessment of Goniotrichum Kützing, Erythrotrichia Areschoug, Diconia Harvey, and Stylonema Reinsch (Rhodophyta). Taxon 34:502-505. 


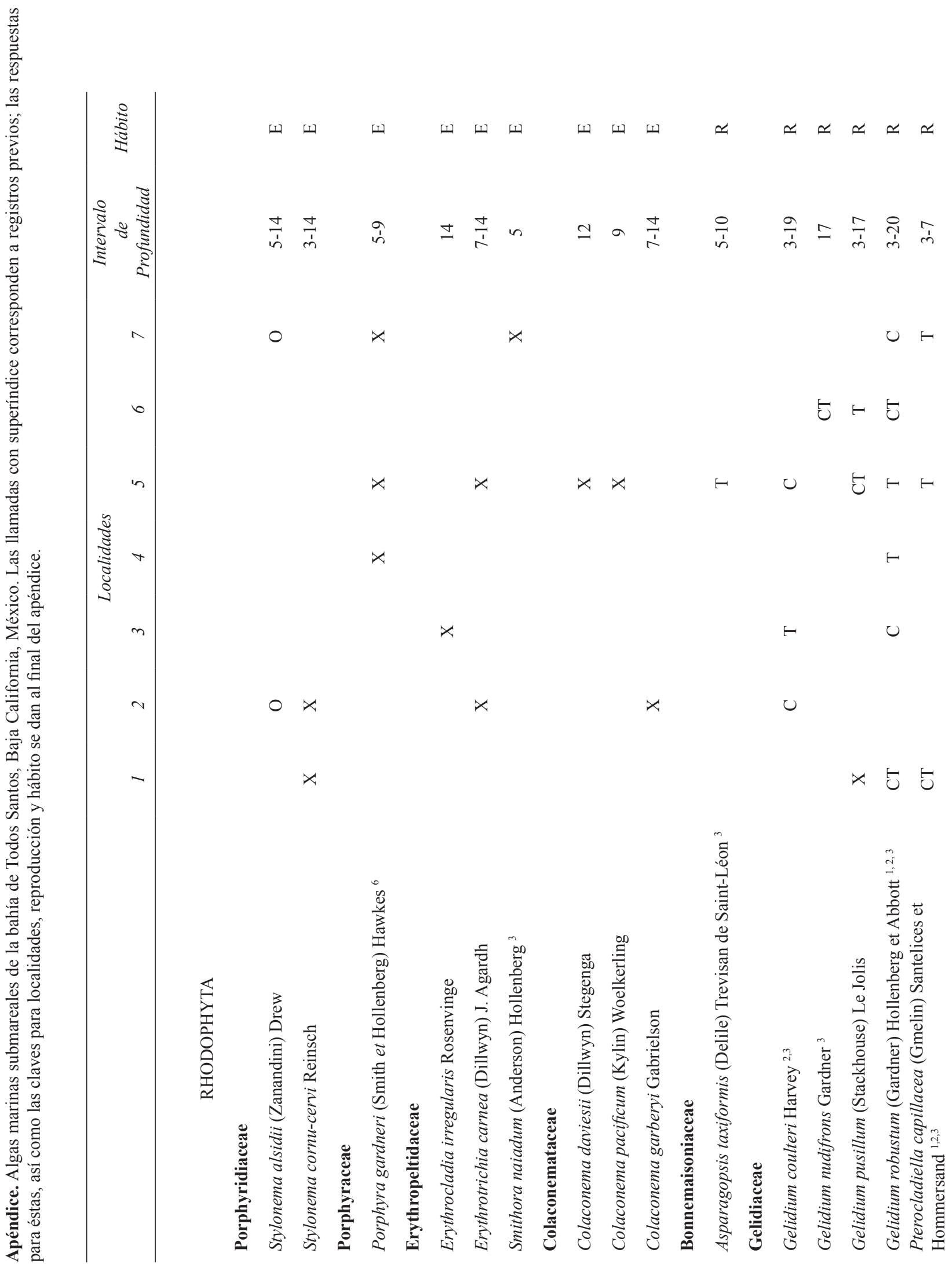




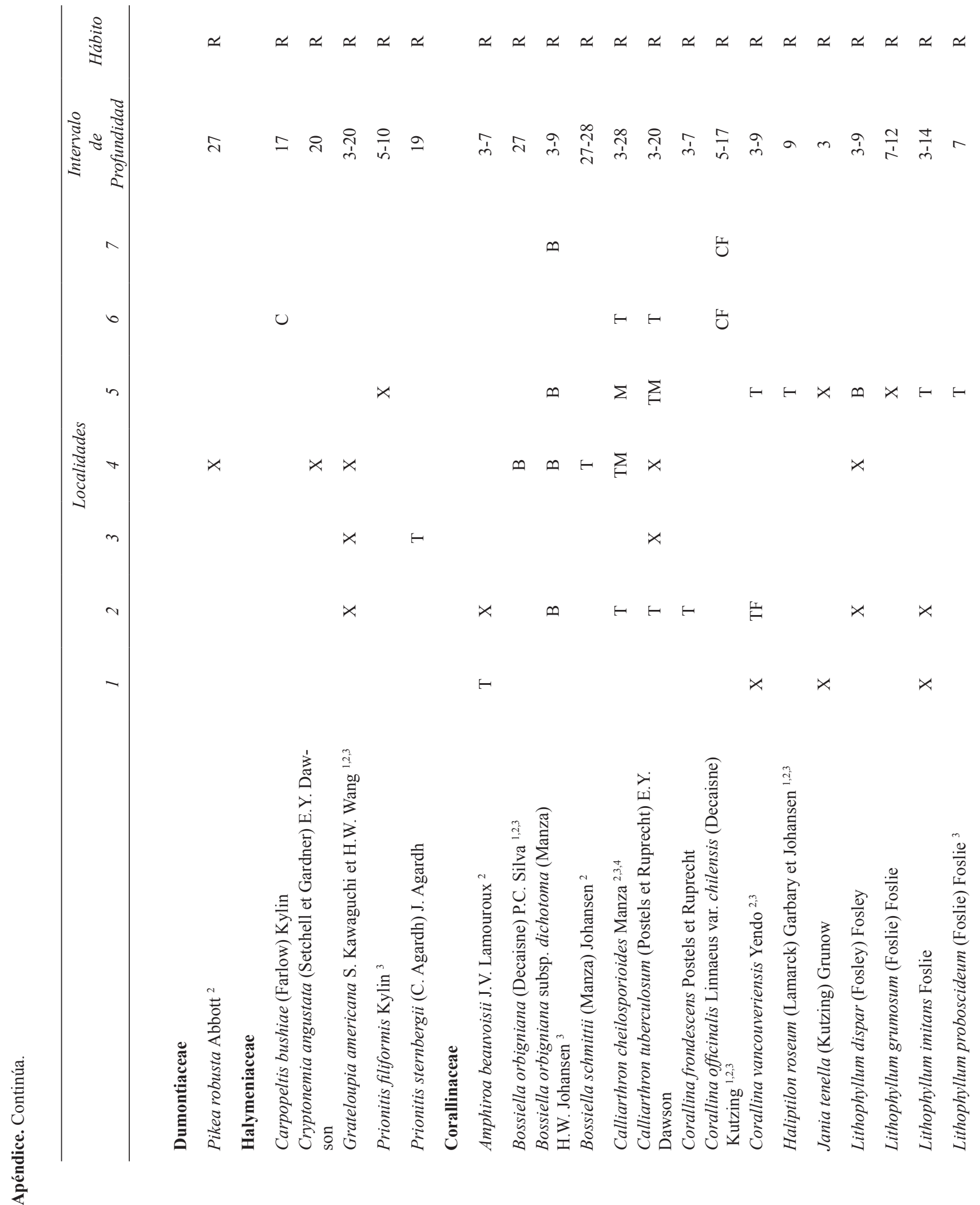




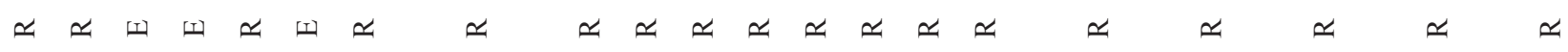

ते

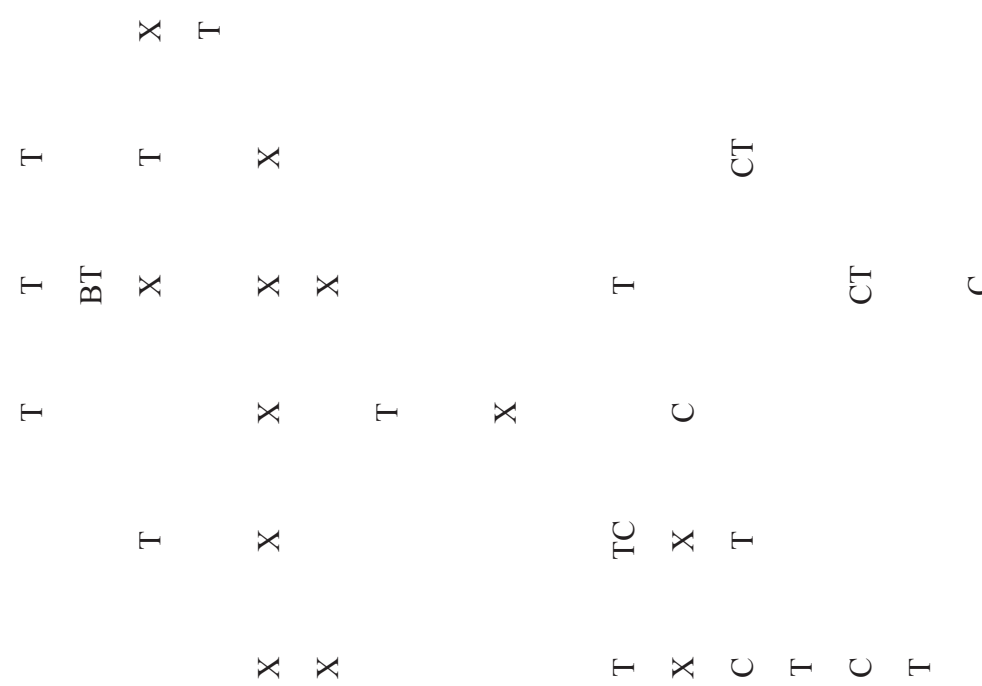

$\mapsto \bullet \times \mapsto$

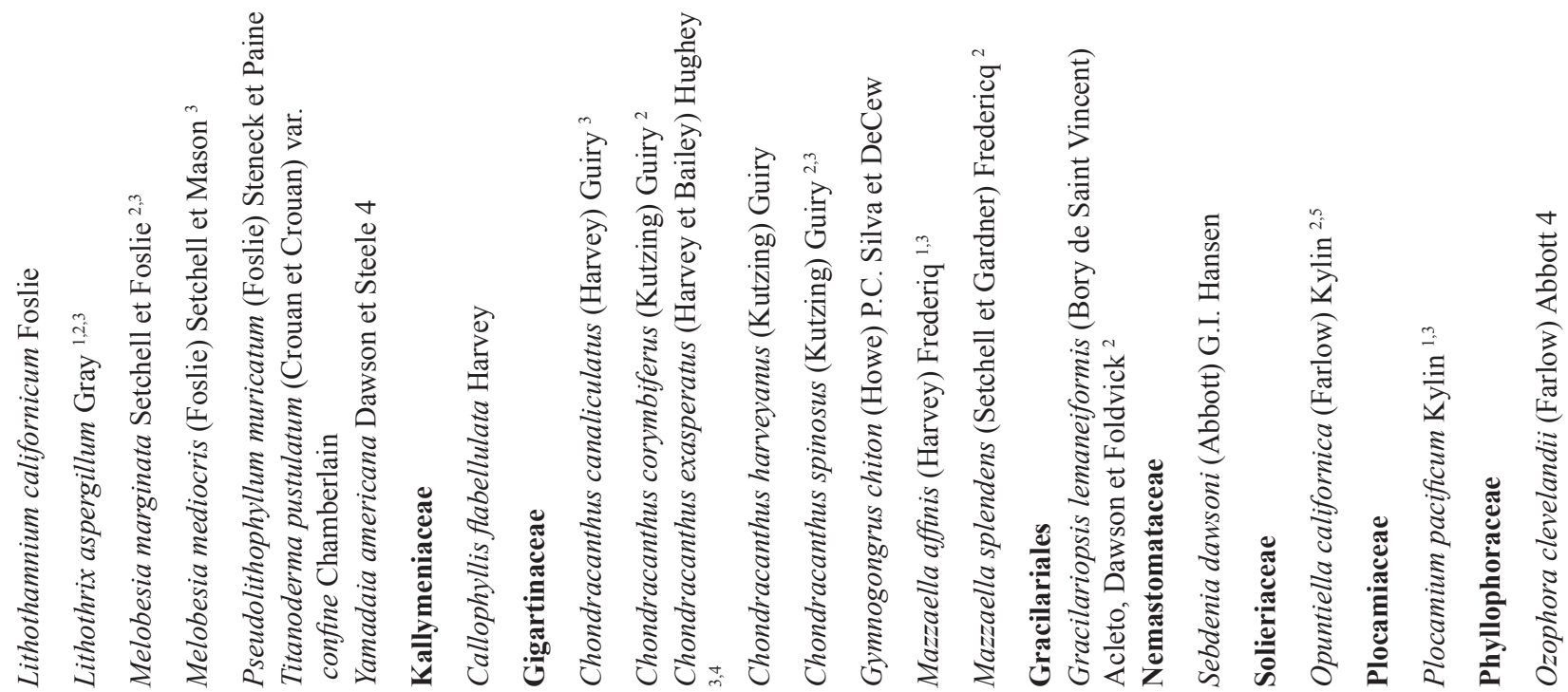




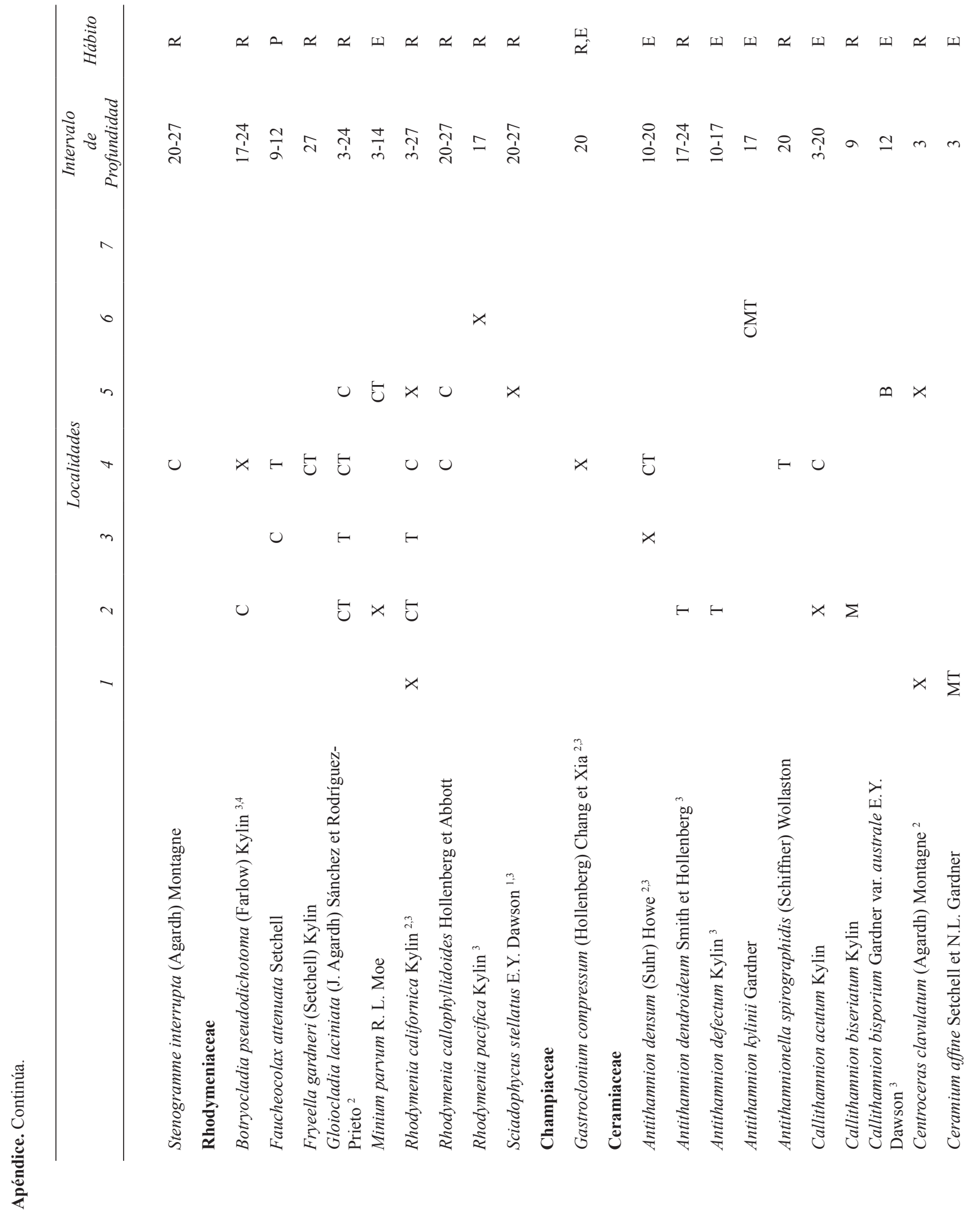




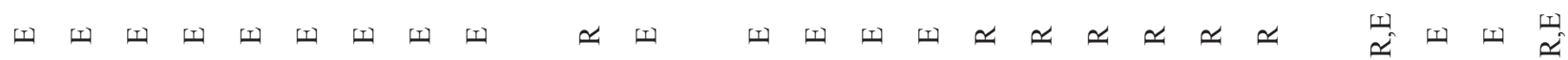

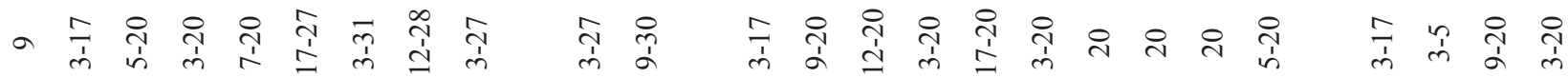

$\mapsto$ 它

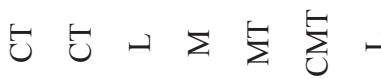
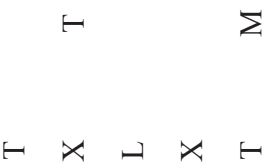

$\sum_{i}^{E} \times x \sum_{j}^{E} x$

-

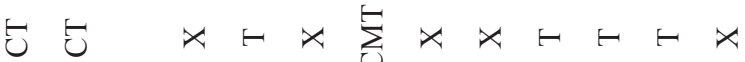

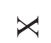

$x$

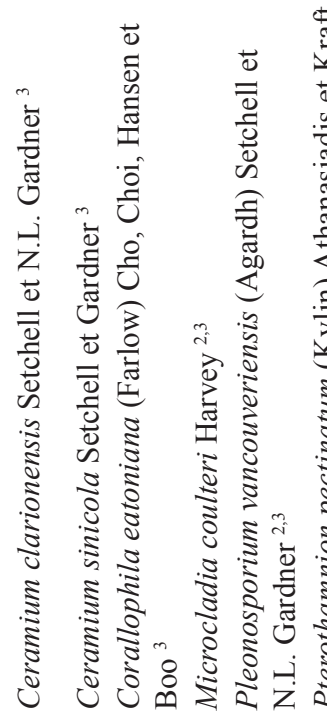

$$
\sum
$$

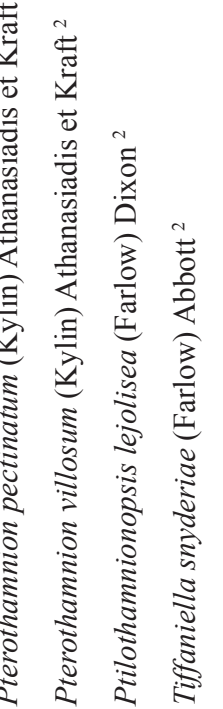

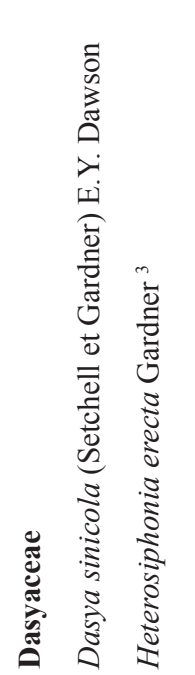

$x x$

$\mapsto$

$\mapsto$

$\times$

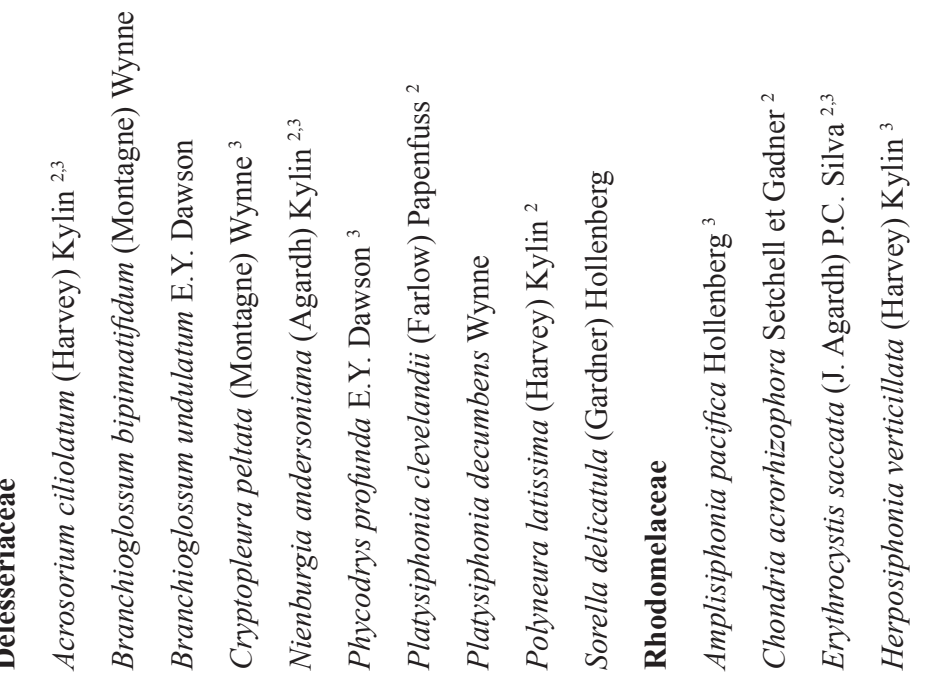


616

Aguilar-Rosas et al.- Macroalgas submareales de Baja California

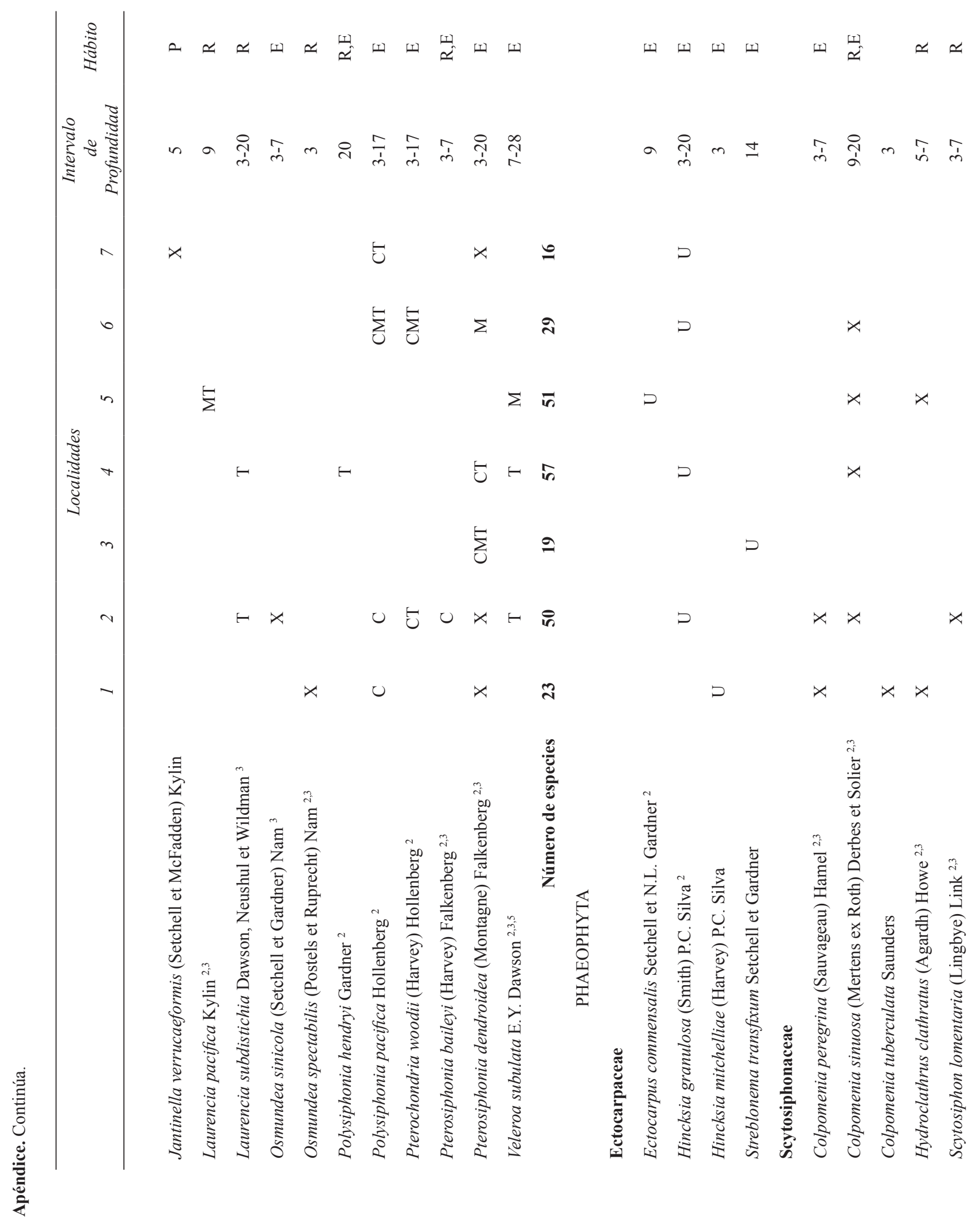


II $\simeq \simeq \simeq \simeq \simeq \simeq \simeq \simeq \simeq \simeq \simeq$

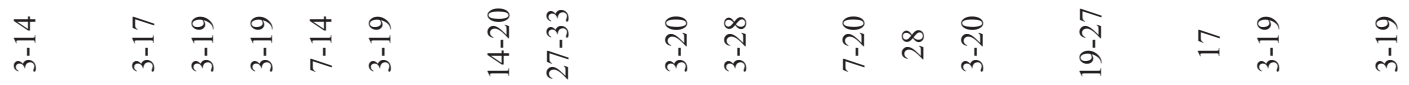

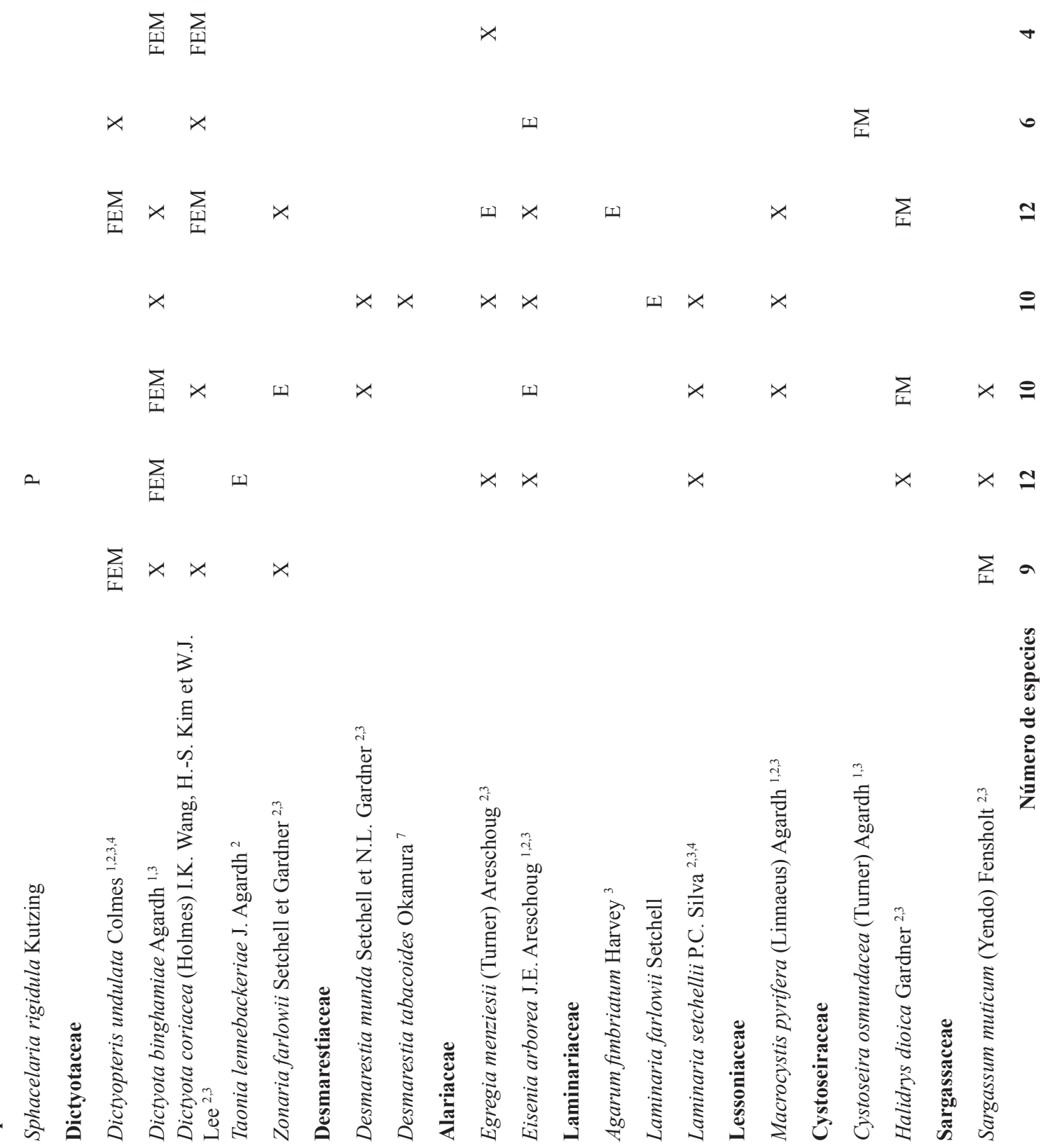




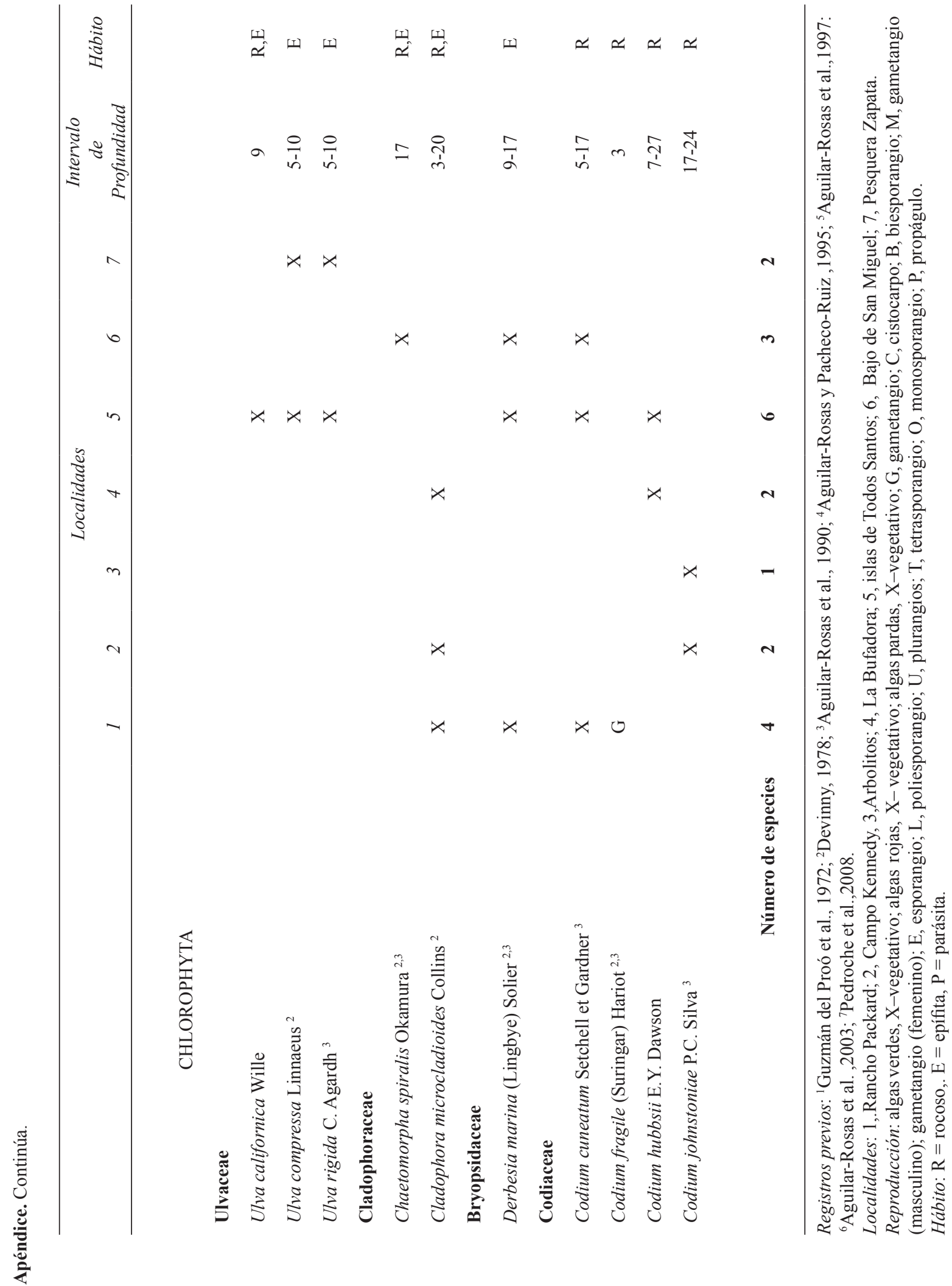

